\title{
Novel Effects in B System: From SUSY to Intrinsic Charm
}

\author{
George Wei-Shu Hou \\ Department of Physics, National Taiwan University, Taipei, Taiwan 10764, R.O.C.
}

\begin{abstract}
We have entered the era of BaBar, Belle and Tevatron competition; with new hardware and unprecedented statistics reach, we must be prepared for discovering new phenomena. While these unfoldings could be coming from new physics, it could also come about as new tricks from old. We illustrate new physics with generic bsg dipole and its impact on $\sin 2 \Phi_{\phi K_{S}}$, and at a deeper level, the marriage of flavor symmetries and SUSY, which could impact on $B_{d}, B_{s}$ and $D^{0}$ mixings and CP violation, and possibility of a light $\widetilde{s b}$ squark. As simple unfolding, we touch upon charmless $B \rightarrow$ baryonic pair decay, with or without an associated $\eta^{\prime} / \gamma$. We close with the possible spectacular signal of $B \rightarrow J / \psi D \pi$ as a flabbergasting new trick from nonperturbative QCD: intrinsic charm of $B$.
\end{abstract}

\section{New Physics Signals: Where Large?}

Traditionally, new physics signals creep out initially as rather faint effects. In the B Factory era (including Tevatron Run II), we pray that new physics would emerge with a splash. We give below three scenarios for flavor violation in context of SUSY.

\subsection{Generic bsg Dipole: $\sin 2 \Phi_{\phi K_{S}} \neq \sin 2 \Phi_{J / \psi K_{S}}$ ?}

It is known that squark-gluino loops can generate sizable $b_{R} \rightarrow{ }^{S} L g$ transitions, which probes a possible new CP phase 1 associated with $b_{R}$ that is not probed by $V_{\mathrm{CKM}}$. Parametrizing the dipole strength as $c_{11}=\left|c_{11}\right| e^{i \sigma}$, the coupling was employed to enhance the direct $\mathrm{CP}$ asymmetries $\left(a_{\mathrm{CP}}\right)$ in $B^{0} \rightarrow K^{+} \pi^{-}$mode, rumored to be sizable in late 1997. By interfering destructively with SM penguins to satisfy $B^{+} \rightarrow \phi K^{+} \lesssim 5 \times 10^{-6}$ from CLEO, it was found ${ }^{3}$ that $a_{\mathrm{CP}}\left(K_{+}^{+} \pi^{-}\right)>50 \%$ is possible. Subsequently, CLEO reported 4 no evidence for $a_{\mathrm{CP}}\left(K^{+} \pi^{-}\right)$. This diminishes, but does not eliminate, the prospects for direct $\mathrm{CP}$ in $\phi K$ mode, especially since Belle discovered 5 that $B^{+} \rightarrow \phi K^{+}$is considerably above the old CLEO bound. Besides $a_{\mathrm{CP}}$, we are now more interested in mixing-dep. $\mathrm{CP}$ in $\phi K_{S}$ mode. Taking as illustration that $b \rightarrow s g \sim 2.5 \%$, which is 10 times larger than $\mathrm{SM}$ but very hard to rule out, we find 6 that $\Phi_{\phi K_{S}}$ could be shifted by $\sim 20^{\circ}$, leading to e.g. $\sin 2 \Phi_{\phi K_{S}} \simeq 0.93$ for $\sin 2 \Phi_{J / \psi K_{S}} \simeq 0.48$ (the Belle value).

\subsection{Generic Abelian Flavor Symmetry with SUSY}

New physics in flavor sector is likely since little is understood. The intriguing pattern of mass and mixing hierarchies in powers of $\lambda \equiv\left|V_{u s}\right|$ suggest

$$
\frac{M_{u}}{m_{t}} \sim\left[\begin{array}{ccc}
\lambda^{7} & \lambda^{5} & \lambda^{3} \\
? & \lambda^{4} & \lambda^{2} \\
? & ? & 1
\end{array}\right], \quad \frac{M_{d}}{m_{b}} \sim\left[\begin{array}{ccc}
\lambda^{4} & \lambda^{3} & \lambda^{3} \\
? & \lambda^{2} & \lambda^{2} \\
? & ? & 1
\end{array}\right]
$$

where the upper right is from $U_{L}, D_{L} \sim V_{\mathrm{CKM}} \equiv U_{L}^{\dagger} D_{L}$ which holds in suitable basis. Note that the lower left are diagonalized by $U_{R}, D_{R}$ but unknown to us with SM dynamics only. Eq. (1) clearly suggest some possible underlying flavor (horizontal) symmetry. If this symmetry is Abelian, commuting horizontal charges imply $M_{i j} M_{j i} \sim M_{i i} M_{j j}(i, j$ not summed $)$, hence

$$
\frac{M_{u}}{m_{t}} \sim\left[\begin{array}{ccc}
\lambda^{7} & \lambda^{5} & \lambda^{3} \\
\lambda^{6} & \lambda^{4} & \lambda^{2} \\
\lambda^{4} & \lambda^{2} & 1
\end{array}\right], \quad \frac{M_{d}}{m_{b}} \sim\left[\begin{array}{ccc}
\lambda^{4} & \lambda^{3} & \lambda^{3} \\
\lambda^{3} & \lambda^{2} & \lambda^{2} \\
\lambda & 1 & 1
\end{array}\right],
$$

is inferred. It is intriguing, then, that $M_{d}^{32} / m_{b}, M_{d}^{31} / m_{b}$ are the most prominent off-diagonal elements, hence impact on $B_{d}$ and $B_{s}$ mixings naturally, iff right-handed down sector can be heard. However, the SM has no righthanded flavor dynamics. This is where SUSY enters to help: $\tilde{d}_{R}$ couples to $\tilde{g}$.

Assuming that SUSY breaking itself does not introduce flavor violations, we find that $\left(\widetilde{M}_{q}^{2}\right)_{L R}^{i j}=\left(\widetilde{M}_{q}^{2}\right)_{R L}^{\dagger} \sim$ $\widetilde{m} M_{d}^{i j},\left(\widetilde{M}_{Q}^{2}\right)_{L L} \sim \widetilde{m}^{2} V_{C K M}$, but

$$
\left(\widetilde{M}_{d}^{2}\right)_{R R} \sim \widetilde{m}^{2}\left[\begin{array}{ccc}
1 & \lambda & \lambda \\
\lambda & 1 & 1 \\
\lambda & 1 & 1
\end{array}\right]
$$

contribute significantly to $B_{d}$ (or $B_{s}$ ) mixings.

Generic flavor symmetry and its breaking can impact on measurables via SUSY! We stress that the flavor and $\mathrm{CP}$ violation in Eq. (3) are on the same footing as $V_{\mathrm{CKM}}$.

\section{$d_{R}-b_{R}$ Mixing: Low $\sin 2 \Phi_{B_{d}}$ and $D^{0}$ Mixing?}

The $R R$ sector could contribute significantly to $B_{d}$ mixing via $\delta_{d R R}^{13} \sim \lambda$ since this is much larger than $V_{t d} \sim \lambda^{3}$. A simple dimensional analysis suggests that $\widetilde{m}, m_{\tilde{g}} \sim$ $M_{W} / \lambda^{2} \sim \mathrm{TeV}$ scale could generate squark-gluino box diagram fontributions that are comparable to SM. We illustrate this observation in Fig. 1, where $\sin 2 \Phi_{B_{d}}$ via $J / \psi K_{S}$ can range from 0.3 to 1 vs $\sin 2 \phi_{1} \simeq 0.75-0.71$ for $\phi_{3}=65^{\circ}-85^{\circ}$ in $\mathrm{SM}$.

Of particular interest is the low $\sin 2 \Phi_{B_{d}} \sim 0.3-$ 0.4 possibility, stated already $\mathrm{O}$ in May 2000 (before 

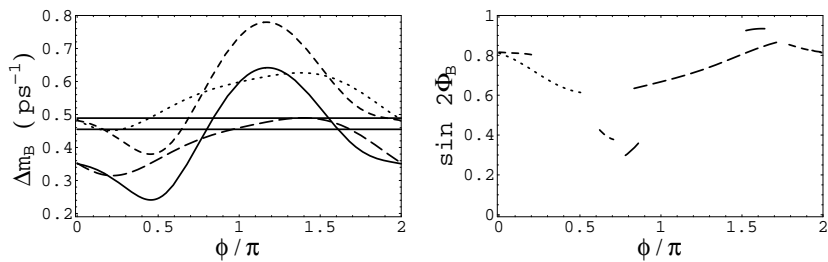

Figure 1: $\Delta m_{B_{d}}$ and $\sin 2 \Phi_{B_{d}}$ vs. $\phi \equiv \arg \delta_{d R R}^{13}$, including both SM and SUSY effects, for squark mass $\widetilde{m}=1.5 \mathrm{TeV}$ (and $\tan \beta=2$ and $\left.|\mu|<m_{\tilde{g}}\right)$. Horizontal double lines indicate $2 \sigma$ experimental range. Solid (short-dash), long-dash (dotted) curves for $m_{\tilde{g}}=1.5$, $3 \mathrm{TeV}$ and $\phi_{3}=65^{\circ}\left(85^{\circ}\right)$, respectively.

ICHEP2000), as compared with the present world average of $0.48 \pm 0.16$, dominated by BaBar $(0.34 \pm 0.20 \pm 0.05)$ and Belle $\left(0.58_{-0.34-0.10}^{+0.32+0.09}\right)$ values reported at this conference. It is clear that CKM unitarity bound from $\Delta m_{B_{s}} / \Delta m_{B_{d}}$ should be relaxed, and potential conflict on $\phi_{3} / \gamma$ w.r.t. charmless rare $B$ decays may be alleviated. What we mean is that, with $\widetilde{m}, m_{\tilde{g}} \gtrsim \mathrm{TeV}$ and $\left(\widetilde{M}_{q}^{2}\right)_{L R, R L}$ suppressed by $m_{q} / \tilde{m}$, there is little impact on penguins, hence charmless rare $B$ decays may have better access to CKM phases (except for hadronic uncertainty). Thus, $\phi_{3} / \gamma \gtrsim 90^{\circ}$ may well be the case, 9 which is strengthened by $\pi^{+} \pi^{-} / K^{+} \pi^{-} \sim 1 / 4$ as reported by CLEO, Belle and now BaBar at this conference.

We eagerly await summer results on $\sin 2 \Phi_{B_{d}} !$

But we have been too naive so far: $\Delta m_{K}$ and $\varepsilon_{K}$ constraints are much more stringent. It is impossible to sustain $\delta_{d L L, R R}^{12} \sim \lambda$, even with $\widetilde{m}, m_{\tilde{g}} \gtrsim \mathrm{TeV}$. Traditionally one employs quark-squark alignment (QSA) to impose "texture zeros" on quark mass matrices, i.e. $M_{d}^{12,21}=0$ hence $D_{L, R}^{12}=0$ or highly suppressed.

In so doing, however, one notices that $D_{L}^{12} \simeq 0 \mathrm{im}$ plies $U_{L}^{12} \sim\left|V_{c d}\right|=\lambda$, which is a general consequence of QSA. Thus, $\tilde{u}_{L}-\tilde{c}_{L}$ mixing $\delta_{u L L}^{12} \sim \lambda$ is sizable, which can generate $D^{0}-\bar{D}^{0}$ mixing, right in the ballpark of recent tantalizing hints from the CLEO and FOCUS experiments, $x_{D} \sim 0.01$. Note that the zeros in Fig. 2 reflect cancellation when different terms have common phase, and shows that $x_{D}$ can be considerably below 0.01 . In any case it is exciting that $D^{0}$ mixing at such levels can be further studied at Belle and BaBar.

There is an interesting subtlety for our choice of $M_{d}^{31} \neq 0$ if one wishes to retain $M_{d}^{23,32}: M_{d}^{12,21}$ would

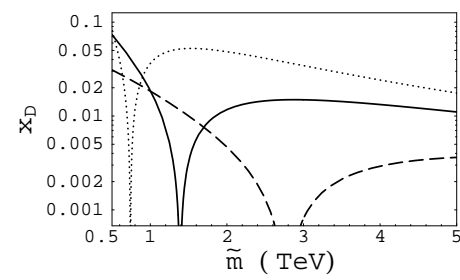

Figure 2: $x_{D}$ vs $\tilde{m}$ for $m_{\tilde{g}}=0.8,1.5$ and $3 \mathrm{TeV}$ and $\tan \beta=2$. once again be generated. Thus, if we choose to keep $\left(\widetilde{M}_{d}^{2}\right)_{R R}^{13} / \widetilde{m}^{2} \sim \lambda$ then $M_{d}^{23}=M_{d}^{32}=0$ need to be imposed on top of $M_{d}^{12}=M_{d}^{21}=0$ and the $s$ flavor is decoupled from $d, b$, hence there will be no new physics effects in $B_{s}$ mixing and $b \rightarrow s \gamma$ decays! We seem to find that the stringent $\Delta m_{K}$ and $\varepsilon_{K}$ constraints imply 4 texture zeros in $M_{d}$. We now turn briefly to the case of decoupling $d$ flavor with QSA. For a more generic discussion of SUSY violation impact on $\sin 2 \Phi_{B_{d}}, B \rightarrow \pi \pi$ and $\rho \gamma$, see the poster talk of C.K. Chua. 1

\section{$s$-b Mixing: $\Delta m_{B_{s}}, \sin 2 \Phi_{B_{s}}$; Light $\widetilde{s b}$ Squark}

I will be brief since this subject is covered by the poster talk of A. Arhrib. 11 The previous $d-b$ mixing case satisfy $\Delta m_{K}, \varepsilon_{K}$ by construction (via alignment), but still have interesting, measurable effects in $B_{d}$ and $D^{0}$ mixings, even if SUSY particles are at TeV scale. The reason is the large $\tilde{d}_{R}-\tilde{b}_{R}$ and $\tilde{u}_{L}-\tilde{c}_{L}$ mixings $(\sim \lambda)$ that arise from Abelian horizontal charges and low energy constraints. Unfortunately, the SUSY scale becomes so high, practically there can be no impact on penguins, hence $\varepsilon^{\prime} / \varepsilon$, $b \rightarrow s \gamma$ and $b \rightarrow d \gamma$ are all unaffected. Though viable, the case is depressing in that squarks and gluino cannot be produced at Tevatron or even the LHC, while there is also no impact on $B_{s}$ System!

Changing the mindset, however, one could have interesting phenomena in a rather similar context: $s-b$ mixing $(\nabla)$ ! Decoupling $d$ flavor now with QSA, one finds,

$$
\left(\widetilde{M}_{d}^{2}\right)_{R R} \sim \widetilde{m}^{2}\left[\begin{array}{lll}
1 & 0 & 0 \\
0 & 1 & 1 \\
0 & 1 & 1
\end{array}\right],
$$

where one has 4 texture zeros analgous to $d$-b case, but $\tilde{s}_{R}-\tilde{b}_{R}$ mixing is $\sim 1$ ! The exciting new feature from the see-saw pattern of Eq. (4) is that, one down type squark, which we call the strange-beauty squark $\widetilde{s b_{1}}$, could be driven light by the large $s-b$ mixing! This is rather different from the scenarios of light stop or sbottom which are generated by large top Yukawa coupling (with or without large $\tan \beta$ ), and represents a third mechanism for having one squark much lighter than the rest, in this case as arising from flavor violation in right-hand sector.

It is truly intriguing that, even for $m_{\widetilde{s b}_{1}}$ and neutralino (dominantly bino) mass $m_{\widetilde{\chi}_{1}^{0}}$ as light as $100 \mathrm{GeV}$, penguins are still little affected, and $b \rightarrow s \gamma$ is quite accommodating 11 even with large $\tilde{s}_{R}-\tilde{b}_{R}$ mixing! But the impact on $B_{s}$ mixing and $\sin 2 \Phi_{B_{s}}$, whether $\widetilde{s} b_{1}$ is light or not, is rather visible, as one can easily see by scaling up from $B_{d}$ result for $d-b$ mixing case. Thus, once again the $\Delta m_{B_{d}} / \Delta m_{B_{s}}$ constraint should be loosened, this time due to $\Delta m_{B_{s}}$ being affected. We note that $D^{0}$ mixing remains volatile and interesting because of alignment. 
If the $\widetilde{s b}_{1}$ is in fact light and with $\widetilde{\chi}_{1}^{0}$ as LSP, there is a change in signature for collider search. Since $\widetilde{s b}_{1}$ has roughly equal mixture of $s$ and $b$ flavor, one should keep in mind that $\widetilde{s} b_{1} \rightarrow b \tilde{\chi}_{1}^{0}, s \tilde{\chi}_{1}^{0}$ are both present, hence $b$ tagging is less efficient. Thus, the direct bound on $\widetilde{s b}$ should be weaker than the standard $\tilde{b}$ squark.

We find with interest that the signatures of $\Delta m_{B_{s}}$, $\sin 2 \Phi_{B_{s}}$ and direct $\widetilde{s b}$ search can all be conducted at the Tevatron Run II, while $D^{0}$ mixing as well as the CKM phase angle pattern can be studied at the B Factories.

\section{Rare Baryons: New Pathways?}

Charmless rare mesonic modes started to emerge in 1997, with many modes now with measured rates $>10^{-5}$. Charmless rare baryonic modes are far less fruitful. We have only the CLEO98 bounds of $B \rightarrow \bar{\Lambda} p, \bar{\Lambda} p \pi^{-}$, $\bar{p} p<0.26,1.3,0.7 \times 10^{-5}$ based on $5.8 \mathrm{M} B \bar{B}$ 's. The corresponding theory is equally sparse: just a handful of models that were "stimulated" by the old ARGUS false observation of $B \rightarrow p \bar{p}(\pi)$ in the late 1980's.

Where is the best place to search?

Observation: Smallness of $B \rightarrow \overline{\mathbf{B}}_{(s)} \mathbf{B}$ likely rooted in the large energy release, aggravated by more complicated composition of baryons ( $q q q)$ vs mesons $(q \bar{q})$. In particular, the 4 -quark operators that mediate $b$ decay quite naturally project a $B$ meson onto a pair of $q \bar{q}$ quarks in final state. Thus, to find larger charmless baryonic $B$ decays, one needs 12 1) reduced energy release and 2) baryonic ingredients in final state.

From these insights, we suggest the natural starting points as: Inclusive $B \rightarrow \eta^{\prime}+X_{s}$ and $\gamma+X_{s}$. Both cases start with large rates, the former $\sim 6 \times 10^{-4}$ for $p_{\eta^{\prime}}>2.0 \mathrm{GeV}$, while the latter $\sim 2 \times 10^{-4}$ for $p_{\gamma} \gtrsim 2.0$ $\mathrm{GeV}$. Both processes have $\eta^{\prime} / \gamma$ carry away large energy, hence reduced energy release in the recoil $X_{s}$ system!

From an inclusive picture of charmless baryon formation, we envision the anomaly mechanism 1 which is effective at spitting out energetic $\eta^{\prime}$ mesons (Fig. 3(a)), followed by $g^{*} \rightarrow$ DD splitting of gluon into diquark pair. In this way, as can be seen from Fig. 3(b), we have baryonic pair ingredients in final state. We then allow a phase space argument for baryon pair formation (Fig. 4).

Since DD pairs already appear to left of $m_{g} \sim 1.1$ $\mathrm{GeV}$ (dots) in Fig. 4, while $\bar{\Lambda} N$ threshold opens up

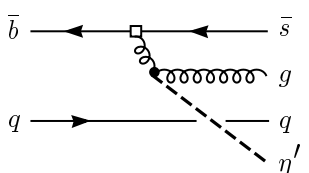

(a)

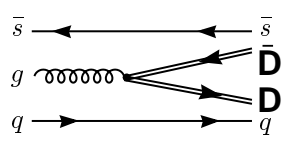

(b)
Figure 3: Anomaly motivated two step process: (a) $g^{*} \rightarrow g^{*} \eta^{\prime}$ mechanism for fast $\eta^{\prime}$ emission; (b) $g^{*} \rightarrow$ DD diquark pair.

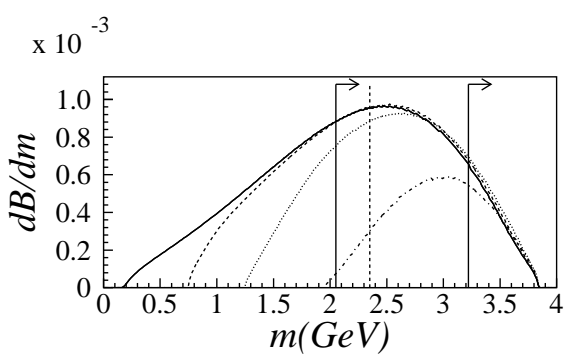

Figure 4: $\quad \bar{B} \rightarrow \eta^{\prime}+s g \bar{q} \rightarrow \eta^{\prime} \mathbf{B}_{(s)} \overline{\mathbf{B}}(\pi)$ from Fig. 3 with solid, dash, dots, dotdash $\sim m_{g}=0,0.6,1.1,1.8 \mathrm{GeV}$ in phase space.

only at $2.05 \mathrm{GeV}$ (left vertical line with arrow), we expect threshold enhancement for $s g \bar{q} \rightarrow \mathbf{B}_{(s)} \overline{\mathbf{B}}(\pi)$ around $m_{X_{s}} \sim 2.3 \mathrm{GeV}$, which corresponds to the experimental cut on $K+n \pi$ partial reconstruction. The modes to search for are $\bar{B} \rightarrow \eta^{\prime} \Lambda \bar{N}$ and similar low lying $\overline{\mathbf{B}}_{s} \mathbf{B}$ states, together with relatively fast $\eta^{\prime}$. Since reconstruction is easy and background is expected to be low $\left(\Lambda_{c}^{+} \bar{N}\right.$ threshold at $3.22 \mathrm{GeV}$ ), the process may offer important probe into higher mass $m_{X_{s}}$ spectrum (the envelope that drops beyond $m_{X_{s}}$ beyond $2.5 \mathrm{GeV}$ ) that is important for confirming the anomaly mechanism itself.

Further encouragement is obtained by improving 13 the pole model approach 12 by making analogy (see Fig. 5 ) of $B \rightarrow \eta^{\prime} p \bar{\Lambda}$ with the recently reported $B \rightarrow D^{*-} p \bar{n}$ mode by CLEO. Assuming factorization, using $B \rightarrow D^{*}$ form factors and incorporating proton form factor $(\mathrm{FF})$, the vector current part can account for $\sim$ half the observed rate, with the other half presumably through axial-vector (e.g. $a_{1}$ ) channel. Extending to $B \rightarrow \eta^{\prime} \bar{\Lambda} p$, $\gamma \bar{\Lambda} p$, perhaps even $\ell \nu \bar{N} N$, we caution that there is no analogy to proton $\mathrm{FF}$, but this may actually imply a larger effect. We therefore suggest 12 that $B \rightarrow \eta^{\prime} \bar{\Lambda} p$, $\gamma \bar{\Lambda} p \sim 10^{-5}>\bar{\Lambda} p$ as plausible, and may be the first charmless baryon mode(s) to be observed. One has the extra bonus of self-analyized spin in $\Lambda \rightarrow p \pi$ decay, which may probe $B \rightarrow \eta^{\prime}, \gamma$ dynamics via the $\mathrm{CP}$ odd and even $\Delta_{\text {odd, even }}=\kappa_{\bar{\Lambda}} \mp \kappa_{\Lambda}$, where $\kappa_{\Lambda}=\boldsymbol{s}_{\Lambda} \cdot\left(\boldsymbol{p}_{p} \times \boldsymbol{p}_{\Lambda}\right.$ and $\kappa_{\bar{\Lambda}}=\boldsymbol{s}_{\bar{\Lambda}} \cdot\left(\boldsymbol{p}_{p} \times \boldsymbol{p}_{\bar{\Lambda}}\right)$ are both T-odd. New physics may be eventually uncovered by such triple products.

Of course, search for traditional $B \rightarrow \overline{\mathbf{B}}_{(s)}$ B 2-body modes should continue! Unlike $K \pi>\pi \pi$, we find that $\bar{B}^{0} \rightarrow \Sigma^{+} \bar{p} \lesssim \bar{B}^{0} \rightarrow p \bar{p}$ as the two leading modes.

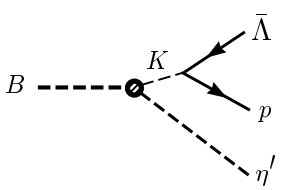

(a)

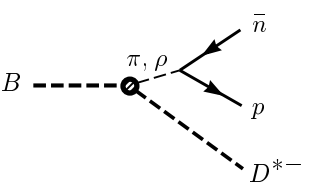

(b)
Figure 5: Analogy between (a) $B \rightarrow \eta^{\prime} p \bar{\Lambda}$ and (b) $B \rightarrow D^{*-} p \bar{n}$ 


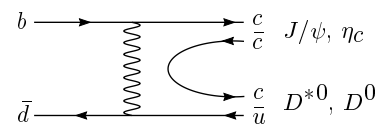

(a)

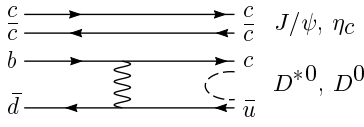

(c)

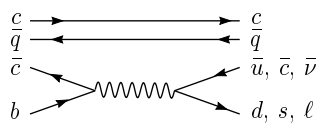

(e)

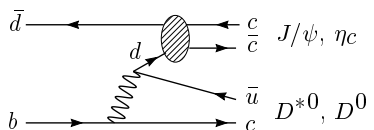

(b)

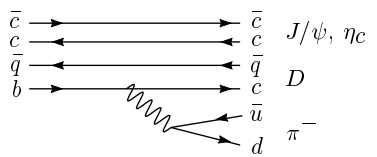

(d)

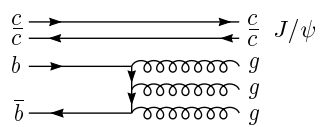

(f)
Figure 6: (a), (b) Suppressed $B \rightarrow J / \psi D$ decay; (c)-(f) $B, B_{c}$ and $\Upsilon$ decay via intrinsice charm Fock component.

3 Intrinsic Charm: $B \rightarrow J / \psi D \pi$ !?

At first sight, this seems like a red herring: 3 charm quarks in $B$ decay final state! Clearly, the exchange and OZI violating processes of Figs. 6(a) and (b) should be extremely suppressed. This could be an advantage, howeyer, depending on what intrinsic charm (IC) could do. 14 Naively, Fig. 6 (c) could lead to $\bar{B}^{0} \rightarrow J / \psi D^{(*) 0}$, but would probably suffer from annihilation suppression, while for Fig. 6(d), normal spectator picture leads to $\bar{B} \rightarrow J / \psi D \pi^{-}$, but is close to the phase space limit. The question is the distribution and strength of IC.

The distributions can be readily obtained, as is shown in Fig. 7. The strength of IC is of nonperturbative origin, and cannot be deduced from first principles yet. An analysis of EMC data indicates that IC of proton could be $\sim 0.86 \%$. Since the $B$ meson is smaller than the proton because of a larger reduced mass, we expect a larger IC fraction in $B$ than in light hadrons! We may therefore hope for IC in $B$ to be greater than $1 \%$.

It is exciting 4 that the above scenario may already have some experimental bearing. Published CLEO data as well as new Belle results from ICHEP2000 hint at an excess of low momentum $J / \psi$ events from $B$ decay. The curves in Fig. 8 are simple fits, with solid for excess below $0.9 \mathrm{GeV}$ assuming $D \pi$ recoil starting at $0.66 \mathrm{GeV}$, the $D^{*}$ recoil threshold. We see that $\bar{B} \rightarrow J / \psi D \pi^{-}$ from Fig. 6(d) provides a plausible explanation for this
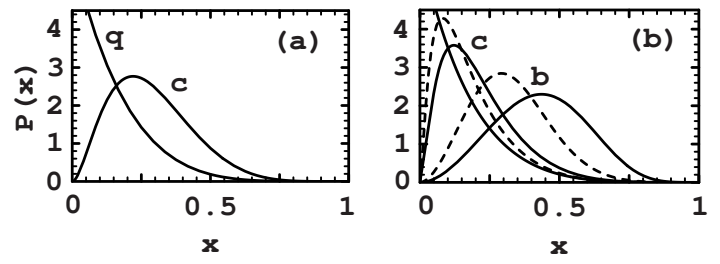

Figure 7: $\mathrm{IC}$ in (a) $p$ and (b) $B$ meson (dashes for $\Upsilon(1 S)$ ).

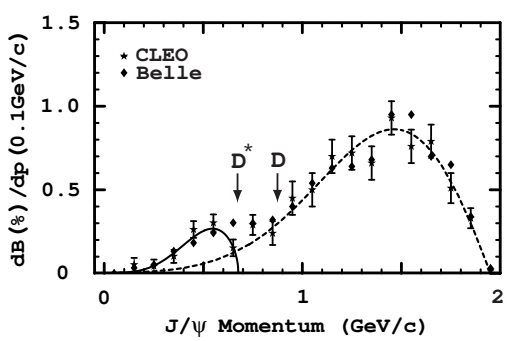

Figure 8: Feed-down subtracted inclusive $B \rightarrow J / \psi+X$.

excess. There may be a hint of $\bar{B} \rightarrow J / \psi D^{*}$, but there is no indication for $J / \psi D$. The plausibility is enhanced when we find that an IC at $1 \%$ level or higher with distribution as indicated in Fig. 7, can account for 14 the rate of few $\times 10^{-4}$. The search should be straightforward, and verification could be as early as this summer.

We note that the process of Fig. 6(f) may explain the soft spectrum of $\Upsilon(1 S) \rightarrow J / \psi+X$, where $p_{J / \psi}$ peak at $\sim 1.5 \mathrm{GeV}$. It would be amusing if smoking gun evidence for IC emerges at B Factories, rather than for lighter hadrons.

Acknowledgement. I have enjoyed collaborating with Abdes Arhrib, Chia-Hung Chang, Chun-Khiang Chua, Amarjit Soni, Shang-Yuu Tsai and Kwei-Chou Yang, as well as earlier collaborators.

\section{References}

1. W.S. Hou and B. Tseng, Phys. Rev. Lett. 80 (1998) 434.

2. T. Bergfeld et al., Phys. Rev. Lett. 81 (1998) 272.

3. X.G. He, W.S. Hou and K.C. Yang, Phys. Rev. Lett. 81 (1998) 5738.

4. S. Chen et al., Phys. Rev. Lett. 85 (2000) 525.

5. P. Chang, in proceedings of ICHEP2000; A. Bozek, this proceedings.

6. W.S. Hou and K.C. Yang, to appear.

7. A. Abashian et al., Phys. Rev. Lett. 86 (2001) 2509; M. Hazumi, this proceedings.

8. C.K. Chua and W.S. Hou, Phys. Rev. Lett. 86 (2001) 2728 [hep-ph/ $\underline{0005015] .}$

9. X.G. He, W.S. Hou and K.C. Yang, Phys. Rev. Lett. 83, 1100 (1999); W.S. Hou, J.G. Smith and F. Würthwein, hep-ex/9910014.

10. C.K. Chua, this proceedings; A. Arhrib, C.K. Chua and W.S. Hou, to appear.

11. A. Arhrib, this proceedings; A. Arhrib, C.K. Chua and W.S. Hou, hep-ph/0103094.

12. W.S. Hou and A. Soni, hep-ph/0008079, to appear in Phys. Rev. Lett.

13. C.K. Chua, W.S. Hou and S.Y. Tsai, to appear.

14. C.H.V. Chang and W.S. Hou, hep-ph/0101162. 